OPEN ACCESS

Edited by:

Siuly Siuly,

Victoria University, Australia

Reviewed by:

Chia Yee Ooi,

Universiti Teknologi, Malaysia

Sławomir Wilczyński,

Medical University of Silesia, Poland

*Correspondence:

Makoto Segawa

segawama@yamaguchi-u.ac.jp

Specialty section:

This article was submitted to

Medtech Data Analytics,

a section of the journal

Frontiers in Medical Technology

Received: 28 October 2021 Accepted: 26 November 2021

Published: 22 December 2021

Citation:

Segawa M, lizuka N, Ogihara $H$,

Tanaka K, Nakae H, Usuku K and Hamamoto $Y$ (2021) Construction of a Standardized Tongue Image Database for Diagnostic Education: Development of a Tongue Diagnosis e-Learning System.

Front. Med. Technol. 3:760542. doi: 10.3389/fmedt.2021.760542

\section{Construction of a Standardized Tongue Image Database for Diagnostic Education: Development of a Tongue Diagnosis e-Learning System}

\author{
Makoto Segawa ${ }^{1 *}$, Norio lizuka ${ }^{1,2}$, Hiroyuki Ogihara ${ }^{3}$, Koichiro Tanaka $^{4}$, Hajime Nakae ${ }^{5}$, \\ Koichiro Usuku ${ }^{6}$ and Yoshihiko Hamamoto ${ }^{3}$ \\ ${ }^{1}$ Department of Kampo Medicine, Yamaguchi University Hospital, Ube, Japan, ${ }^{2}$ Yamaguchi Health Examination Center, \\ Yamaguchi, Japan, ${ }^{3}$ Division of Electrical, Electronic and Information Engineering, Graduate School of Sciences and \\ Technology for Innovation, Yamaguchi University, Ube, Japan, ${ }^{4}$ Department of Traditional Medicine, Faculty of Medicine, \\ Toho University, Tokyo, Japan, ${ }^{5}$ Department of Emergency and Critical Care Medicine, Akita University Graduate School of \\ Medicine, Akita, Japan, ${ }^{6}$ Department of Medical Information Science and Administrative Planning, Kumamoto University \\ Hospital, Kumamoto, Japan
}

Tongue examination is an important diagnostic method for judging pathological conditions in Kampo (traditional Japanese medicine), but it is not easy for beginners to learn the diagnostic technique. One reason is that there are few objective diagnostic criteria for tongue examination findings, and the educational method for tongue examination is not standardized in Japan, warranting the need for a tongue image database for e-learning systems that could dramatically improve the efficiency of education. Therefore, we constructed a database comprising tongue images whose findings were determined on the basis of votes given by five Kampo medicine specialists (KMSs) and confirmed the educational usefulness of the database for tongue diagnosis e-learning systems. The study was conducted in the following five steps: development of a tongue imaging collection system, collection of tongue images, evaluation and annotation of tongue images, development of a tongue diagnosis e-learning system, and verification of the educational usefulness of this system. Five KMSs evaluated the tongue images obtained from 125 participants in the following eight aspects: (i) tongue body size, (ii) tongue body color, (iii) tongue body dryness and wetness, (iv) tooth marks on the edge of the tongue, (v) cracks on the surface of the tongue, (vi) thickness of tongue coating, (vii) color of tongue coating, and (viii) dryness and wetness of tongue coating. Medical students (MSs) were given a tongue diagnosis test using an e-learning system after a lecture on tongue diagnosis. The cumulative and individual match rates (\%) (individual match rates of 100\% (5/5), 80\% (4/5), and 60\% (3/5) are shown in parentheses, respectively) were as follows: (i) tongue body size: 92.8 (26.4/26.4/40.0); (ii) tongue body color: 83.2 (10.4/20.8/52.0); (iii) tongue body dryness and wetness: 88.8 (13.6/34.4/40.8); (iv) tooth marks on the edge of the tongue: 88.8 (6.4/35.2/47.2); (v) cracks on the surface of the tongue: 96.8 (24.0/35.2/37.6); (vi) thickness of tongue coating: 84.8 (7.2/21.6/56.0); (vii) color of tongue coating: 88.0 (15.2/37.6/35.2); and 
(viii) dryness and wetness of tongue coating: 74.4 (4.8/19.2/50.4). The test showed that the tongue diagnosis ability of MSs who attended a lecture on tongue diagnosis was almost the same as that of KMSs. We successfully constructed a tongue image database standardized for training specialists on tongue diagnosis and confirmed the educational usefulness of the e-learning system using a database. This database will contribute to the standardization and popularization of Kampo education.

Keywords: tongue examination, tongue diagnosis, database, e-learning, Kampo, education, malnutrition, glossitis

\section{INTRODUCTION}

Since tongue findings reflect a variety of systemic disorders, including malnutrition, tongue examination is one of the basic physical examinations. Median rhomboid glossitis, atrophic glossitis, lichen planus, leukoplakia, hairy tongue, and glossodynia are sometimes associated with tongue disorders. Morphological abnormalities, including geographic tongue, fissured tongue, and macroglossia, are also often observed (1-3). These are associated with various pathological conditions, including malnutrition, infectious diseases, systemic diseases, hereditary diseases, age-related changes, and unknown causes (13). In particular, atrophic glossitis is associated with malnutrition (4) and is accompanied by a smooth red tongue surface with atrophy of the tongue papilla. Suppression of tongue papilla cell regeneration is caused by insufficient intake or malabsorption of specific nutrients such as iron (Plummer-Vinson syndrome due to iron deficiency anemia) (5), vitamin B12 (pernicious anemia) (6), vitamin B2, and folic acid (7).

Tongue findings are also important in Kampo medicine, which is the traditional Japanese medicine. It is based on traditional Chinese medicine but developed as a unique form in Japan. Kampo has been the backbone of Japanese medicine for more than 1,500 years (8-10). Historically, observation of the tongue as a diagnostic methodology first appeared in China around the Jin (1115-1234) and Yuan Dynasties. The extant first work of tongue diagnosis is the Ao Shi Shang Han Jin Jing Lu (Ao's Records of Golden Mirror on Cold Pathogenic Diseases) written in 1341. It was later brought to Japan and published in 1654. Tongue examination was deeply studied during the Edo period (1603-1868) in Japan $(11,12)$.

Kampo medicine doctors consider the physical and mental conditions comprehensively through four examinations, i.e., inspection, listening and smelling examination, medical interview, and examination by touch manipulation, to determine the final diagnostic pathology, "sho," and administer Kampo medication $(13,14)$. Tongue diagnoses were included in the inspection. Tongue findings are considered to reflect physical and mental conditions. The doctor evaluates the color, morphology, and movement of the tongue body, and the color, morphology, and dryness of the tongue coating, and determines their association with an imbalance of Ki (Qi) (vital life force energy), Ketsu (blood), and Sui (body fluid). Kampo

Abbreviations: ERs, engineering researchers; KMS, Kampo medicine specialists; MSs, medical students; SD, standard deviation. diagnosis is divided into four dichotomic categories:Yin/You (yin/yang), Kyo/Jitsu (deficiency/excess), Kan/Netsu (cold/heat), and Hyou/Ri (exterior/interior) (13-15).

The normal color of the tongue body is defined as light red in Kampo medicine. Compared with normal color, pale, red, deep red, and purple tongues indicate deficiency and cold, heat, advanced heat, and blood stasis, respectively. Normal morphology of the tongue body is defined as a tongue without swelling, atrophy, tooth marks, or cracks. Compared with normal morphology, swelling, atrophy, tooth marks, and cracks indicate water retention and $\mathrm{Ki}(q \mathrm{i})$ deficiency, Ki (Qi) deficiency and Ketsu deficiency, Sui retention and/or Ki (Qi) deficiency, and Ketsu deficiency and/or lack of Sui, respectively $(16,17)$.

In a healthy person, the tongue is kept clean by the action of moistening and cleaning by saliva, chewing movement, mechanical friction of the tongue movement, normal oral flora, and nutritional supply. However, if these functions are impaired, the hygienic environment in the oral cavity deteriorates, and the tongue coating tends to increase. The tongue coating is composed of exfoliated cells, mucus, bacteria, and food residue. A thin white tongue coating is considered normal, while a thick white coating suggests cold, yellow coating suggests heat, and no coating suggests both $\mathrm{Ki}(\mathrm{Qi})$ and Ketsu deficiency. Dry tongue coating suggests yang state, while wet tongue coating suggests the yin state in Yin-Yang theory $(16,17)$.

As mentioned above, tongue diagnosis is a basic examination technique in Kampo medicine; however, it is not easy for beginners to master it. To successfully offer a tongue diagnosis to patients, long-term training in distinguishing various complex tongue findings and understanding pathological conditions is essential. Furthermore, the lack of standard diagnostic criteria hinders accurate tongue diagnosis, which consequently depends on the subjective evaluation of the practitioner. The ambiguity of tongue diagnosis may inhibit the development of Kampo medicine research and the implementation of standardized Kampo medicine education. Therefore, there is a need to develop a modern teaching strategy for the efficient and accurate education of tongue diagnosis techniques. To solve the aforementioned problems, we constructed a database of standard tongue images that could guarantee diagnostic quality and developed a tongue diagnosis electronic learning (e-learning) system. Furthermore, we have proposed an objective method determined by a majority vote to ensure the reliability of the diagnostic information of the tongue images used in the database. The educational advantage of this system is that it can be learned at any place and time repeatedly, and the diagnostic ability of 
the tongue can be evaluated. The introduction of this learning system is expected to dramatically improve the learning efficiency of tongue diagnosis and promote standardization in tongue diagnosis education.

This research was jointly conducted by KMS and engineering researchers (ERs) who are familiar with educational technology and image recognition. The aims of this study were to construct a database of tongue images, which are annotated with correct labels by KMSs, and to create a tongue diagnosis e-learning system using the database and verify its educational usefulness.

\section{MATERIALS AND METHODS}

This study was conducted in the following sequence: Step 1: Development of tongue image capture and collection system; Step 2: Collection of tongue image data; Step 3: Evaluation and annotation of tongue image; Step 4: Development of a tongue diagnosis e-learning system; and Step 5: Evaluation of the educational usefulness of this system. This study was evaluated from a scientific and ethical point of view in accordance with the Declaration of Helsinki and the ethical guidelines for medical and health research for humans established by the Ministry of Health, Labor and Welfare of Japan and approved by the ethical review committee at the Clinical Research Center of Yamaguchi University Hospital (Research Approval Number: H27-067-3).

\section{Step 1: Development of Tongue Imaging Collection System}

While taking pictures, environmental factors, such as the color tone and illuminance of the lighting, the quality of the shooting device, the shooting method, and the way the subject's tongue sticks out, could affect the image quality. To obtain clear tongue images suitable for interpretation, we developed a tongue image capture and collection system (inventor: Yoshihiko Hamamoto, Norio Iizuka, et al. Japanese Patent Application Laid-Open No. 2019-213652), which can constantly adjust the shooting environment. This system is a tablet-type mobile device with a camera function. The feature of this system is that a tongueshaped framework is set on the filming screen on the tablet to capture the tongue of the subject. When filming the tongue according to the framework, the filming conditions are naturally unified; thus, biases due to the difference in the size and direction of the tongue and the method of tongue presentation can be reduced. As the filming distance between the subject and the camera is kept constant, defocusing can also be prevented (Figure 1). The tongue images were taken using a tablet-type device (ASUS ZenPad ${ }^{\mathrm{TM}}$ 8.0 Z380M, screen resolution: 1,280 $\times 800$ pixels, ASUSTeK Computer Inc.). Regarding lighting, one arm-type desk lamp (MCST-13K, illuminance: 300 lux, fluorescent lamp type: FPL13EX, MAXER DENKI Co., Ltd.) was placed on both diagonal sides of the patient. The color of the fluorescent lamp was "daylight," which is a whitish light close to natural light. The illuminance in the room where the image was taken was measured with a digital illuminator (TM-205, Tenmars electronics co. ltd.), and we confirmed that the illuminance was maintained at $\sim 800(745-865)$ lux.

\section{Step 2: Collection of Tongue Image Data}

Using the tongue imaging collection system, we acquired tongue image data of 125 patients who visited the Kampo Medicine Outpatient Department of Yamaguchi University Hospital (100 patients) and St. Hill Hospital (25 patients). Written informed consent was obtained from all the participants. The inclusion criteria were as follows: (1) patients who visited the Kampo medicine outpatient department of Yamaguchi University Hospital and St. Hill Hospital, (2) patients aged 20 years or older, and (3) patients who obtained written consent voluntarily to participate in the study. Exclusion criteria were patients who did not meet the above inclusion criteria. The data collection period lasted from December 2019 to July 2020. There were 36 men and 89 women in the samples; the patients' ages ranged from 24 to 93 years $(60.7 \pm 15.0$ years $)$.

The photographs were taken in a room with an appropriate illuminance of $700-800 \mathrm{~lx}$, with auxiliary lighting devices attached to both sides of the subject. The image was taken within $10 \mathrm{~s}$ of the presentation of the tongue. Shootings were repeated three times independently, and the image data were saved in the computer as anonymized data. A researcher other than the photographer selected the best quality tongue images.

\section{Step 3: Evaluation and Annotation of Tongue Images}

In general, a diagnosis given by only one doctor could be unreliable. If the same diagnosis given by multiple doctors, it indicates high concordance, and the reliability can be regarded as high. In addition, when specialists with vast clinical experience give a diagnosis, its reliability increases. Therefore, in this study, five KMSs with vast clinical experience diagnosed the same tongue images on the same level of display using laptop computers with same specifications at different locations. If the same diagnostic result was given by three or more of the five KMSs, we defined it as the correct diagnosis (the correct answer).

They evaluated eight findings from 125 tongue images: (i) tongue body size, (ii) tongue body color, (iii) tongue body dryness and wetness, (iv) tooth marks on the edge of the tongue, (v) cracks on the surface of the tongue, (vi) thickness of tongue coating, (vii) color of tongue coating, and (viii) dryness and wetness of tongue coating. Individual match rates of 5/4/3 evaluators and cumulative match rates were calculated for each of the eight items. In addition, the diagnostic results for each of the eight items were analyzed.

The diagnosis of the tongue images by five KMSs was performed using the same computer with the same specifications equipped with software that allowed each evaluator to input the diagnostic result. To ensure anonymity and objectivity, the diagnostic data were analyzed by the ERs.

All the five KMSs were proficient in Kampo practice and certified by the Japan Society for Oriental Medicine. The average age of the KMSs (48-62 years old) was $55 \pm 5.5$ years old [mean \pm standard deviation (SD)], and each one belonged to a different medical institution.

Since the tongue diagnosis results were confirmed by majority vote of the KMSs, the results were high-quality information, 

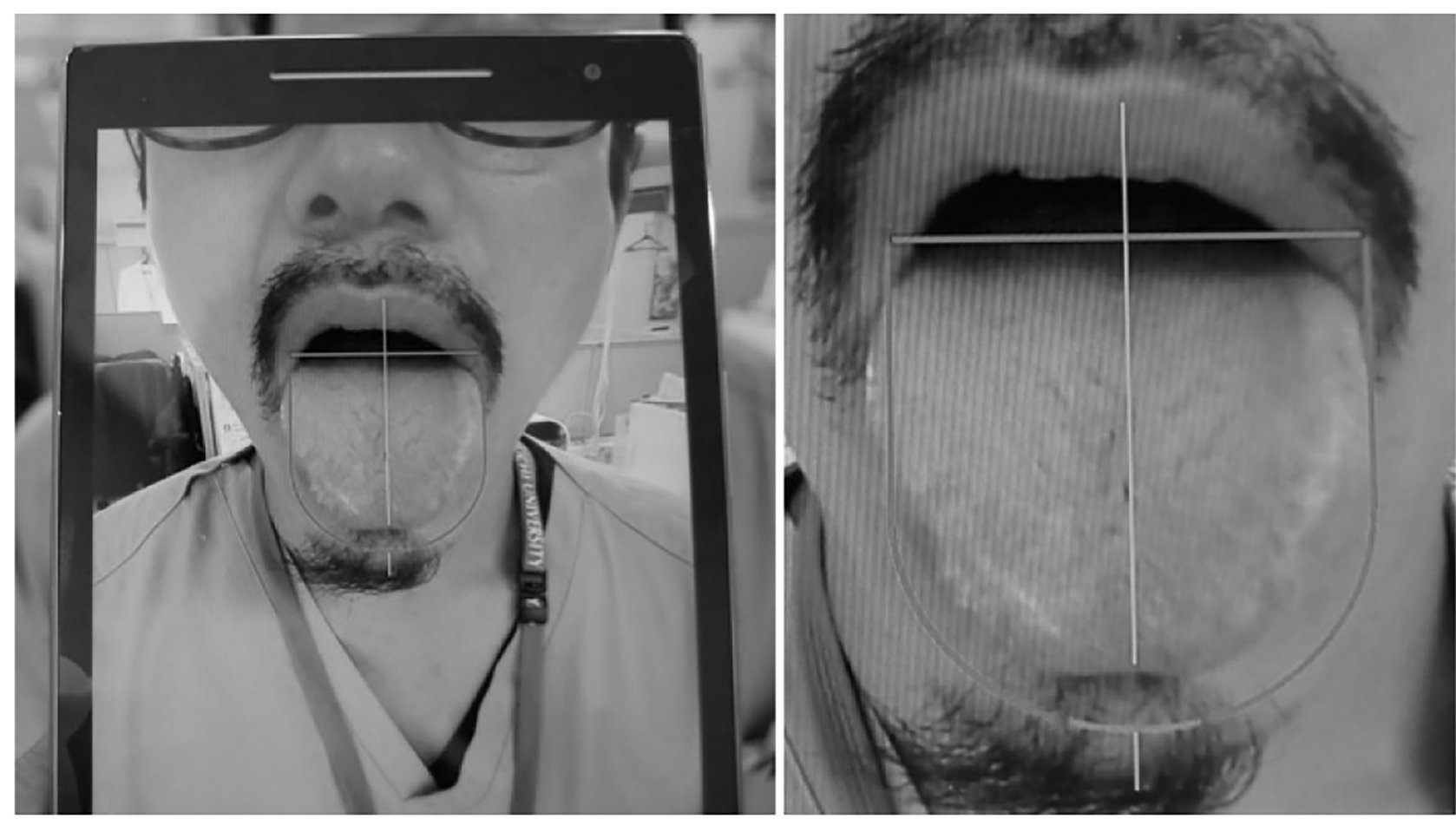

FIGURE 1 | Tablet-type mobile device equipped with a tongue image collection system. Tablet-type mobile device with a camera function that has a "tongue-shaped framework" set on the shooting screen. When shooting the tongue according to the framework, the shooting conditions are naturally unified; thus, the shooting bias due to the difference in the size and direction of the tongue and the manner of tongue presentation can be reduced.

ensuring reliability and objectivity. A tongue image with a high diagnostic match rate was considered a high-quality image showing the typical characteristics of each item and suitable for educational use. In this way, a tongue image database with a high-quality, correct labels was constructed.

\section{Step 4: Development of Tongue Diagnosis e-Learning System}

We developed a tongue diagnosis e-learning system that utilizes images from a quality-guaranteed tongue image database. This e-learning system makes it possible to not only learn without restrictions on time, place, and equipment but also perform a self-evaluation of tongue diagnostic ability. The procedure is as follows: (1) access an e-learning website; (2) pre-learning: confirm the eight standard tongue images displayed on their device before taking the test (Figure 2); (3) tongue diagnosis test: questions about the presented tongue image are shown in Figure 3; (4) send answer data and check grades: immediately after sending the answer data, check the score, correct/incorrect display of all questions, and 8-items radar chart; (5) confirmation of deviation value in the test group: When taking the test in a group, the average value, maximum score, minimum score, own score, frequency distribution, and radar chart of the group are displayed. This allows the learners to see their grades in a group.

\section{Step 5: Verification of the Educational Usefulness of Tongue Diagnosis e-Learning System}

To evaluate the educational usefulness of this learning system, we created a test of 80 questions (eight findings in the tongue images obtained from 10 participants) (Table 1; Figure 4), which was administered to 112 MSs of Yamaguchi University School of Medicine after they received a lecture on tongue diagnosis. The five KMSs took the same test without a lecture. The MSs took the test with a smartphone. From the student's answer data, the average score, score distribution, and correct answer rate for each of the eight items were calculated, and the degree of acquisition of tongue diagnosis ability was evaluated. The answers of the MSs were compared with those of the five KMSs.

\section{Statistical Analysis}

Values are expressed as the mean \pm SD. The significance of differences was determined using the Student's $t$-test. (PASW ${ }^{\circledR}$ Statistics 18). Statistical significance was set at $P \leq 0.05$.

\section{Description of Medical Terms in Kampo Medicine}

The Three Substance Categories: Ki, Ketsu, and Sui

$\mathrm{Ki}(\mathrm{Qi})$ is the vital force energy that is the source of movement of the human body, which moves blood, water, and organs, and regulates the mental state, autonomic nervous system, endocrine 


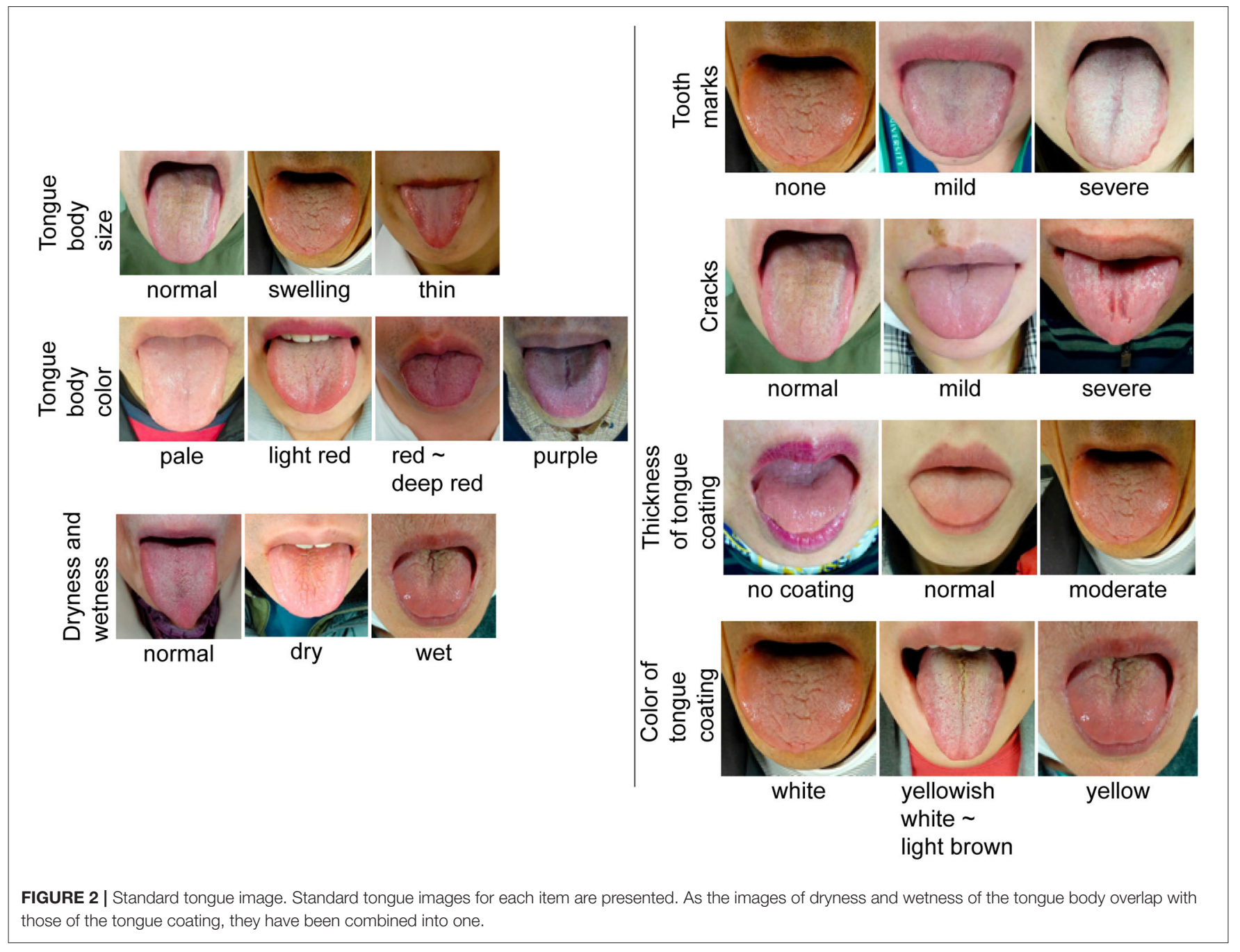

system, and immune system. Ketsu (blood) refers to the red fluid in the body and is considered a vital nutrient substance, which nourishes bodily tissues and organs and ensures adequate mental function. Sui (body fluids) refers to the clear fluids in the body, which moisten and nourish the body and includes saliva, gastric juices, joint fluids, tears, mucus, sweat, and urine. A state in which $\mathrm{Ki}$, Ketsu, and Sui circulate in a well-balanced manner is considered healthy, and if any of them is excessive, insufficient, or stagnant, illness will occur (13-15).

\section{Disease Condition Categories: Yin-You, Kyo-Jitsu, Kan-Netsu, and Hyou-Ri}

The four dichotomic categories are used to classify the patient's disease condition or state and determine the Sho, which means symptoms, signs, or evidence. If the repairing responses shown by the patient against his/her disease condition are feverish, active, and excitatory, the patient is said to be in You-Sho (positive or active pattern). Conversely, if the responses shown by the patient are chilly, inactive, and inhibitory, the patient is said to be in Yin-Sho (negative or passive pattern). Further, if the repairing responses shown by the patient against his/her disease condition are strong or fully active, the patient is said to be in Jitsu-Sho (excess pattern), while if they are weak or hollow, he/she is said to be in Kyo-Sho (deficiency pattern). If the repairing responses shown by the patient against his/her disease condition are febrile, the patient is said to be in Netsu-Sho (heat pattern), while if they are chilly, the patient is said to be in Kan-Sho (cold pattern). If the part where the reaction against the disease appears is near the body surface, it is called Hyou-Sho (exterior pattern), but if it is deep inside the body, it is called Ri-Sho (interior pattern) $(13,14)$.

\section{RESULT}

\section{Match Rate of Tongue Diagnosis Results of Five KMSs}

The match rate of the tongue diagnosis results of the five KMSs for 125 tongue images was calculated for each item (Table 2). The cumulative match rate (\%) (cumulative match rate of three or more evaluators) and individual diagnosis match rate (\%) (match rates of 5/4/3 evaluators are shown in parentheses) for each of the eight items are as follows: (i) tongue body size: 92.8 


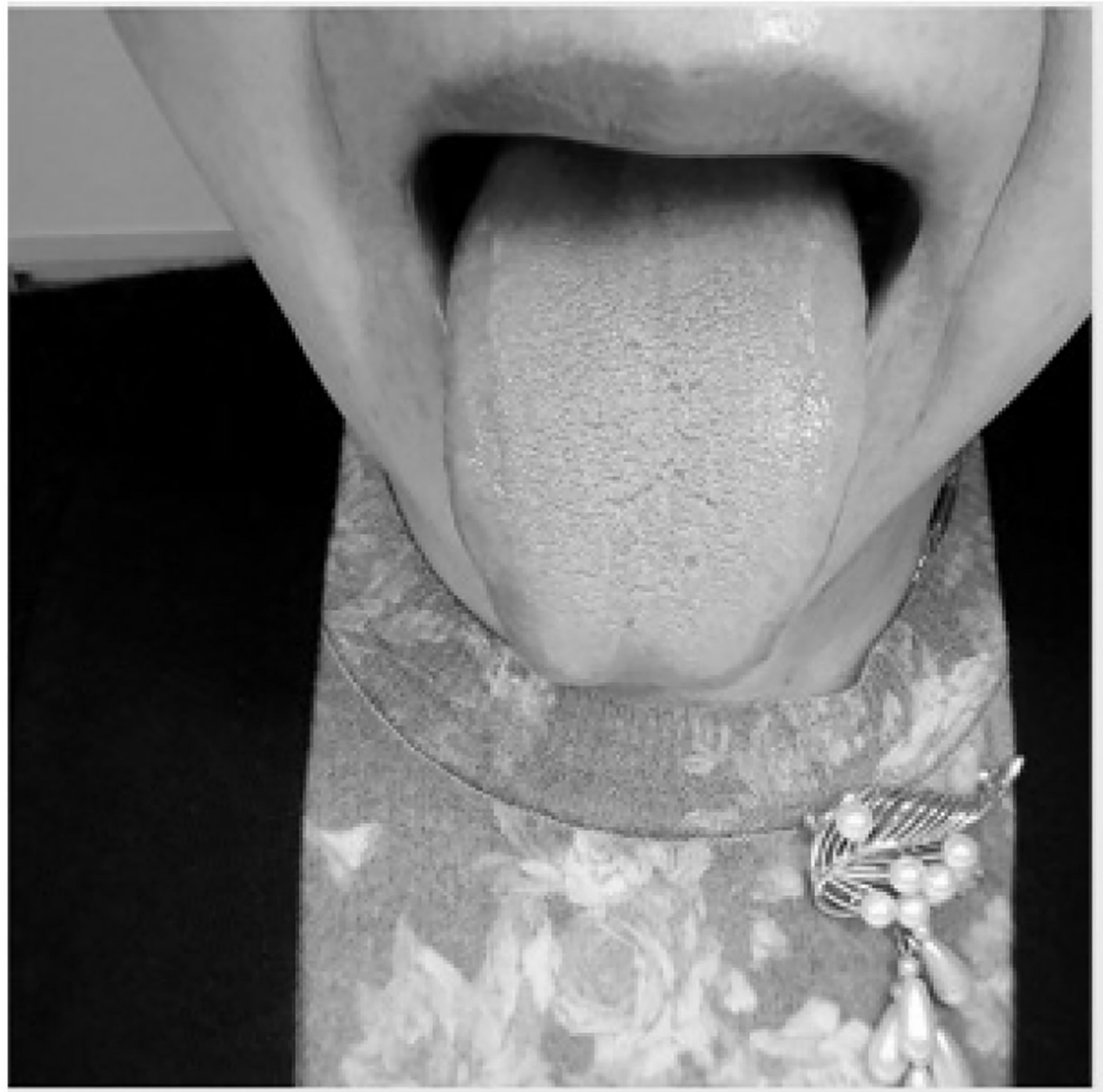

In the tongue image shown above, select the tongue color.
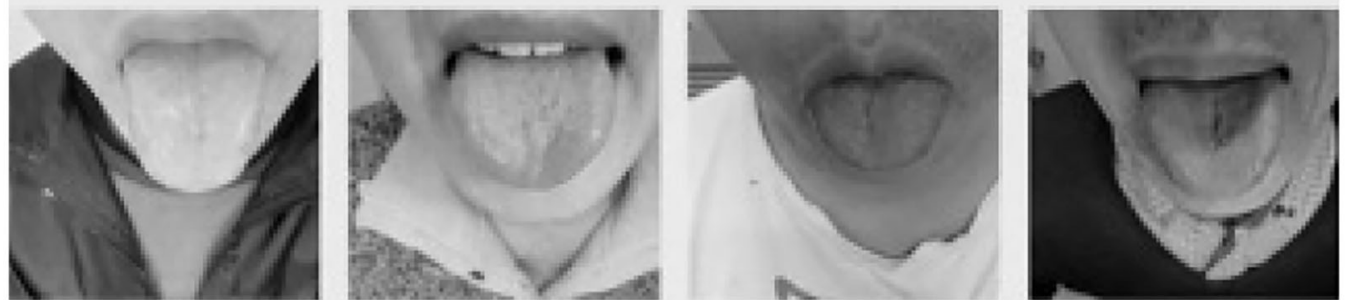

Example image: Pale, light red, red to deep red, purple in order from left to right. Choose one from the options below.

1. pale

2. light red

3. red $\sim$ deep red

4. purple

5. not applicable 
TABLE 1 | Correct answer of the 80 questions used for the tongue diagnosis test.

\begin{tabular}{|c|c|c|c|c|c|c|c|c|c|c|}
\hline & Image 1 & Image 2 & Image 3 & Image 4 & Image 5 & Image 6 & Image 7 & Image 8 & Image 9 & Image 10 \\
\hline Tongue body size & Normal & Normal & Normal & Swelling & Swelling & Normal & Thin & Normal & Normal & Swelling \\
\hline Tongue body color & Light red & Pale & Red-dark red & Purple & Light red & Light red & Light red & Purple & Red-dark red & Light red \\
\hline $\begin{array}{l}\text { Tongue body dryness and } \\
\text { wetness }\end{array}$ & Normal & Normal & Wet & Normal & Normal & Normal & Normal & Dry & Normal & Normal \\
\hline Tooth marks & None & None & None & None & Mild & None & None & None & None & None \\
\hline Cracks & None & None & Mild & Mild & None & None & None & Mild & Mild & None \\
\hline Thickness of tongue coating & Normal & Normal & Normal & Normal & Normal & Normal & Normal & Moderate & Moderate & $\begin{array}{l}\text { Moderate or } \\
\text { higher with } \\
\text { some peeling }\end{array}$ \\
\hline Color of tongue coating & White & White & White & $\begin{array}{l}\text { Yellowish } \\
\text { white-light } \\
\text { brown }\end{array}$ & White & White & White & $\begin{array}{l}\text { Yellowish } \\
\text { white-light } \\
\text { brown }\end{array}$ & White & $\begin{array}{l}\text { Yellowish } \\
\text { white-light } \\
\text { brown }\end{array}$ \\
\hline $\begin{array}{l}\text { Dryness and wetness of } \\
\text { tongue coating }\end{array}$ & Normal & Normal & Wet & Normal & Normal & Normal & Normal & Wet & Normal & Wet \\
\hline
\end{tabular}

The correct answer was automatically determined by a majority vote of 5 KMSs. KMS, Kampo medicine specialists.

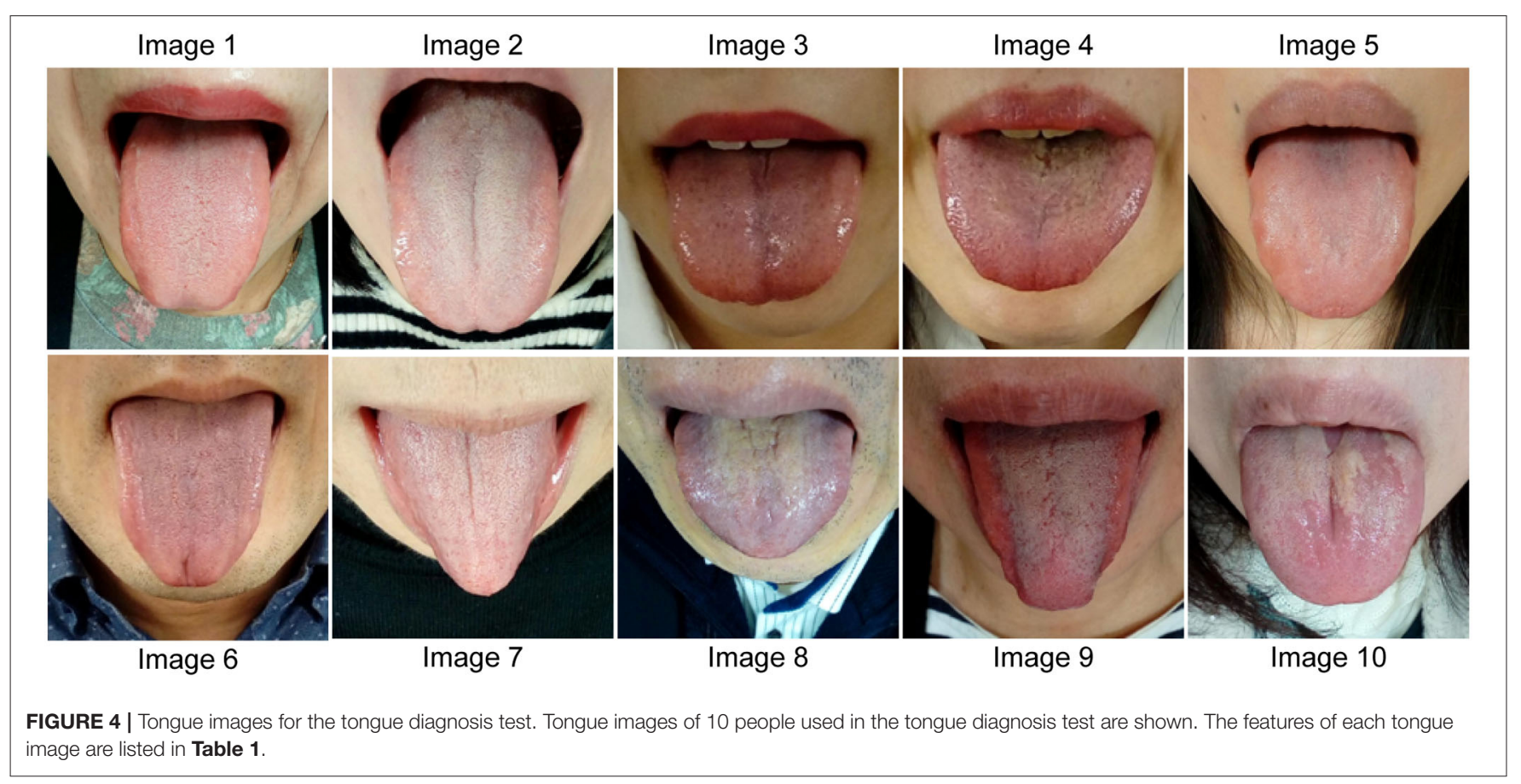

(26.4/26.4/40.0); (ii) tongue body color: $83.2(10.4 / 20.8 / 52.0)$; (iii) tongue body dryness and wetness: 88.9 (13.6/34.4/40.8); (iv) tooth marks on the edge of the tongue: $88.8(6.4 / 35.2 / 47.2)$; (v) cracks on the surface of the tongue: $96.8(24.0 / 35.2 / 37.6)$; vi) thickness of tongue coating: 84.8 (7.2/21.6/56.0); (vii) color of tongue coating: 88.0 (15.2/37.6/35.2); and (viii) dryness and wetness of tongue coating: $74.4(4.8 / 19.2 / 50.4)$. The mean values ( \pm standard deviation) of match rates of $5 / 4 / 3$ evaluators were $13.5 \pm 8.1 \%, 28.8 \pm 7.6 \%$, and $44.9 \pm 7.5 \%$, respectively.

The cumulative diagnostic concordance rate for three or more of the five patients was highest for cracks on the surface of the tongue at $96.8 \%$, lowest for dryness and wetness of tongue coating at $74.4 \%$, and the average value for all the items was $87.2 \pm 6.7 \%$.
The cumulative diagnostic concordance rate for four or more of the five patients was the highest for cracks on the surface of the tongue at $59.2 \%$, the lowest for dryness and wetness of tongue coating at $24.0 \%$, and the average value for all the items was 42.3 $\pm 15.7 \%$.

\section{Analysis of the Diagnosis Results for Each of the Eight Items by Five KMSs}

Images with matching tongue diagnosis results for three or more of the five KMSs were analyzed for each of the eight items in the 125 tongue images (Table 3). (i) Size of the tongue: $56.9 \%$ for normal, $37.1 \%$ for swelling, and $6 \%$ for thin; (ii) color of the tongue: $18.3 \%$ for pale red, $52.9 \%$ for light red, $13.5 \%$ for 
TABLE 2 | Diagnostic match rates of five KMSs.

\begin{tabular}{|c|c|c|c|c|}
\hline & \multicolumn{3}{|c|}{ Individual match rates (\%) } & \multirow{2}{*}{$\begin{array}{c}\begin{array}{c}\text { Cumulative } \\
\text { match rates }(\%) \\
60 \% \text { or more }\end{array}\end{array}$} \\
\hline & $\begin{array}{c}100 \% \\
(5 / 5)\end{array}$ & $\begin{array}{l}80 \% \\
(4 / 5)\end{array}$ & $\begin{array}{l}60 \% \\
(3 / 5)\end{array}$ & \\
\hline Tongue body size & 26.4 & 26.4 & 40.0 & 92.8 \\
\hline Tongue body color & 10.4 & 20.8 & 52.0 & 83.2 \\
\hline $\begin{array}{l}\text { Dryness and wetness of } \\
\text { tongue body }\end{array}$ & 13.6 & 34.4 & 40.8 & 88.8 \\
\hline $\begin{array}{l}\text { Tooth marks on the edge } \\
\text { of the tongue }\end{array}$ & 6.4 & 35.2 & 47.2 & 88.8 \\
\hline $\begin{array}{l}\text { Cracks on the surface of } \\
\text { the tongue }\end{array}$ & 24.0 & 35.2 & 37.6 & 96.8 \\
\hline $\begin{array}{l}\text { Thickness of tongue } \\
\text { coating }\end{array}$ & 7.2 & 21.6 & 56.0 & 84.8 \\
\hline Color of tongue coating & 15.2 & 37.6 & 35.2 & 88.0 \\
\hline $\begin{array}{l}\text { Dryness and wetness of } \\
\text { tongue coating }\end{array}$ & 4.8 & 19.2 & 50.4 & 74.4 \\
\hline Mean value $\pm S D$ & $13.5 \pm 8.1$ & $28.8 \pm 7.6$ & $44.9 \pm 7.5$ & $87.2 \pm 6.7$ \\
\hline
\end{tabular}

The match rate of the tongue diagnosis results of the five KMSs with respect to the tongue images of 125 patients was calculated for each of the eight items. The individual match rate shows the match rate of five out of five KMSs, four out of five KMSs, and three out of five KMSs. The cumulative match rate shows the sum of the individual match rates of three out of five KMSs. KMS, Kampo medicine specialists; $S D$, standard deviation.

red to deep red, and $15.4 \%$ for purple; (iii) dryness and wetness of the tongue body: $58.6 \%$ for normal, $17.1 \%$ for dryness, and $24.3 \%$ for wetness; (iv) tooth marks: $66.7 \%$ for none, $26.1 \%$ for mild, and $7.2 \%$ for severe; (v) cracks: $45.5 \%$ for normal, $44.6 \%$ for mild, and $9.9 \%$ for severe; (vi) thickness of the tongue coating: $1.9 \%$ for none, $49.1 \%$ for normal, $44.3 \%$ for moderate, $3.8 \%$ for moderate or higher with some peeling, and $0.9 \%$ for thick; (vii) color of the tongue coating: $58.2 \%$ for white, $35.5 \%$ for yellow to light brown, 5.5\% for yellow, and $0 \%$ for dark brown to black; (viii) dryness of the tongue coating: $44.1 \%$ for normal, $23.7 \%$ for dryness, and $32.3 \%$ for wetness. In this study, about half of the patients exhibited normal color and morphology, and the other half showed pathological findings.

\section{Evaluation of Diagnostic Consistency of Five KMSs}

To evaluate the consistency of diagnoses of the five KMSs, the responses to the 80 -question test after database construction were compared with those reported at the time of database construction, and the consistency rate was calculated. The average match rates for the eight items were as follows: (i) tongue coating size: $74.0 \pm 16.7 \%$; (ii) tongue coating color: $58.0 \pm$ 19.2\%; (iii) tongue coating dryness and wetness: $68.0 \pm 20.5 \%$; (iv) tooth marks on the edge of the tongue: $70.0 \pm 29.2 \%$; (v) cracks on the surface of the tongue: $64.0 \pm 15.2 \%$; (vi) thickness of tongue coating: $58.0 \pm 19.2 \%$; (vii) color of tongue coating: $46.0 \pm 20.7 \%$; and (viii) dryness and wetness of tongue coating: $72.0 \pm 17.9 \%$. The consistency on the color of the tongue body and color of the tongue coating tended to be low. The average consistency rate for all items was $63.8 \pm 10.1 \%$.
TABLE 3 | Diagnosis result of tongue image determined by majority vote of three or more out of five KMSs.

\begin{tabular}{|c|c|c|c|}
\hline & $\begin{array}{l}\text { Diagnosis } \\
\text { result }\end{array}$ & $\begin{array}{c}\text { Number of } \\
\text { patients }\end{array}$ & Ratio (\%) \\
\hline \multirow[t]{4}{*}{ Tongue body size } & Thin & 7 & 6.0 \\
\hline & Normal & 66 & 56.9 \\
\hline & Swelling & 43 & 37.1 \\
\hline & Total & 116 & 100 \\
\hline \multirow[t]{5}{*}{ Tongue body color } & Pale & 19 & 18.3 \\
\hline & Light red & 55 & 52.9 \\
\hline & $\begin{array}{l}\text { Red or deep } \\
\text { red }\end{array}$ & 14 & 13.5 \\
\hline & Purple & 16 & 15.4 \\
\hline & Total & 104 & 100 \\
\hline \multirow{4}{*}{$\begin{array}{l}\text { Tongue body dryness and } \\
\text { wetness }\end{array}$} & Normal & 65 & 58.6 \\
\hline & Dry & 19 & 17.1 \\
\hline & Wet & 27 & 24.3 \\
\hline & Total & 111 & 100 \\
\hline \multirow{4}{*}{$\begin{array}{l}\text { Tooth marks on the edge of the } \\
\text { tongue }\end{array}$} & None & 74 & 66.7 \\
\hline & Mild & 29 & 26.1 \\
\hline & Severe & 8 & 7.2 \\
\hline & Total & 111 & 100 \\
\hline \multirow{4}{*}{$\begin{array}{l}\text { Cracks on the surface of the } \\
\text { tongue }\end{array}$} & Normal & 55 & 45.5 \\
\hline & Mild & 54 & 44.6 \\
\hline & Severe & 12 & 9.9 \\
\hline & Total & 121 & 100 \\
\hline \multirow[t]{6}{*}{ Thickness of tongue coating } & None & 2 & 1.9 \\
\hline & Normal & 52 & 49.1 \\
\hline & Moderate & 47 & 44.3 \\
\hline & $\begin{array}{l}\text { Moderate or } \\
\text { higher with } \\
\text { peeling }\end{array}$ & 4 & 3.8 \\
\hline & Thick & 1 & 0.9 \\
\hline & Total & 106 & 100 \\
\hline \multirow[t]{6}{*}{ Color of tongue coating } & White & 64 & 58.2 \\
\hline & $\begin{array}{l}\text { Yellowish } \\
\text { white to } \\
\text { brown }\end{array}$ & 39 & 35.5 \\
\hline & Yellow & 6 & 5.5 \\
\hline & $\begin{array}{l}\text { Dark brown } \\
\text { to black }\end{array}$ & 0 & 0 \\
\hline & $\begin{array}{l}\text { Not } \\
\text { applicable }\end{array}$ & 1 & 0.9 \\
\hline & Total & 110 & 100 \\
\hline \multirow{4}{*}{$\begin{array}{l}\text { Dryness and wetness of tongue } \\
\text { coating }\end{array}$} & Normal & 41 & 44.1 \\
\hline & Dry & 22 & 23.7 \\
\hline & Wet & 30 & 32.3 \\
\hline & Total & 93 & 100 \\
\hline
\end{tabular}

The diagnosis results of the tongue image determined by a majority vote of three or more out of five KMSs were analyzed for each of the eight items. Tongue images that were not decided by majority vote were excluded. KMS, Kampo medicine specialists. 


\section{Evaluation of Learning Effect Using e-Learning System \\ Tongue Diagnosis Test Score}

The scores of the tongue diagnosis test of 112 MSs showed a normal distribution from the lowest score of 34 points to the highest score of 100 points (Figure 5). The average score was 60.9 \pm 10.7 points. In contrast, the average score of the five KMSs was $57.5 \pm 12.8$. There was no statistically significant difference between the two scores $(p=0.239)$. Assuming that a score of 60 points or more was used as the criterion for acquiring tongue diagnostic ability, it was considered that $46.4 \%$ of the MSs were able to acquire it.

\section{Comparison of Diagnostic Match Rates Between MDs and KMSs}

Table 4 shows the average diagnostic match rate for 10 tongue images for each of the eight MS and KMS items. The diagnostic match rate of the MSs was similar to that of KMSs, and no statistically significant difference was observed in any of the eight items. The diagnostic match rates (\%) of MSs and KMSs in the eight items are as follows: (i) tongue body size: $62.9 \pm 15.2,68.0$ \pm 23.4 ( $p=0.57$ ), (ii) tongue body color: $65.1 \pm 32.6 \%, 60.0 \pm$ $28.2(p=0.71)$, (iii) tongue body dryness and wetness: $65.3 \pm$ $16.1,66.0 \pm 23.2(p=0.94)$, (iv) tooth marks on the edge of the tongue: $68.9 \pm 19.6,60.0 \pm 28.3(p=0.43),(v)$ cracks on the surface of the tongue: $53.0 \pm 22.9,64.0 \pm 32.4(p=0.39)$, (vi) thickness of the tongue coating: $59.4 \pm 20.7,51.0 \pm 25.1(p=$ 0.43 ), (vii) color of tongue coating: $59.1 \pm 24.6,42.0 \pm 25.7(p$ $=0.15$ ), and (viii) dryness and wetness of tongue coating: $54.5 \pm$ $19.3,50.0 \pm 27.1(p=0.57)$.

\section{DISCUSSION}

Tongue diagnosis in Kampo involves accurately interpreting complex and diverse tongue findings and appropriately identifying Kampo medical pathology indicated by those findings. Although tongue diagnosis is an important technique in Kampo medicine, it has the following problems: (i) there are few diagnostic criteria, (ii) it depends on a subjective evaluation, and (iii) the diagnosis result varies depending on the diagnostic criteria $(18,19)$. This makes it difficult to measure the tongue morphology and color of the patients. In addition, different criteria are used by individual medical doctors who determine the final diagnosis on the basis of tongue findings. These results are inconsistent in terms of diagnosis among medical doctors. We speculate that some of the reasons for the variability in diagnostic results are due to the lack of standardization of diagnostic and training methods in tongue diagnosis.

Recently, standardization of diagnostic methods for tongue examination in Kampo has been attempted $(20,21)$. Standardized educational programs for tongue diagnosis are also important in modern Kampo medical education. Currently, basic education in Kampo medicine is provided in all medical universities in Japan (22). However, little research has been conducted on educational methods for tongue diagnosis, and only a few research studies have been conducted in China $(23,24)$. The traditional learning method of tongue diagnosis is to learn by observing the patient's tongue in daily clinical practice, but it takes a long time and effort to acquire this ability. Therefore, there is a need to develop a modern teaching strategy for the efficient and accurate education of tongue diagnosis techniques. To solve the above problems, we developed a standardized database of tongue images and proposed the utilization of a tongue diagnosis e-learning system.

This new system is underlined by the following concepts: (i) Simplification, visualization, and iterative learning of knowledge necessary for learning tongue diagnosis skills and (ii) management of learning level using an e-learning system. The first concept is to train the image interpretation ability by dividing it into eight fields. By training for the reading and interpretation ability of individual components, it becomes possible to make integrated judgments quickly without much effort. Using this method, the learner can intuitively grasp the judgment criteria of the color and morphology, which are difficult to evaluate quantitatively, and it can improve the discriminating ability. Second, this is a learning management system that uses e-learning. E-learning enables twoway communication via the Internet. The advantages are as follows: (i) a large number of learners can study repeatedly at any time and place; (ii) the quality of education is uniform and not influenced by the quality of the instructor; (iii) inclusion of rare cases; (iv) able to centrally manage the progress of learning and feedback and objectively evaluate the degree of learning. These features are not found in conventional learning methods and are extremely useful not only because the learning content can be standardized and streamlined but also the diagnostic ability, which is the learning goal, can be evaluated.

In recent years, many studies on tongue diagnosis, such as tongue diagnostic equipment (25-27), automatic diagnostic system using artificial intelligence (28-30), and remote diagnosis using smartphones (31), have been conducted. The diagnostic ability by computers is rapidly improving, and the feasibility of standardization of tongue diagnosis using machines has been sought (32). However, little research has been conducted on educational methods and equipment to improve the ability and skill of human tongue diagnosis. Recently, the usefulness of flipped classroom using an e-learning program in Kampo education has been reported (33). In addition, it should be noted that we previously conducted a WEB-based test for medical students to evaluate the learning effect of Kampo medicine in collaboration with several medical universities and reported its usefulness in learning management using information and communication technology $(34,35)$.

In this study, the percentages of images that matched for $5 / 4 / 3$ KMSs were $13.5,28.8$, and $44.9 \%$, respectively. These results suggest that there are some differences in the diagnostic ability of the KMSs. It is presumed that in daily clinical practice, there are a few tongues with classical findings, as shown in a textbook. Additionally, this result might be due, in part, to an individual's ability to discriminate and understand tongue colors. In this study, the average match rate for tongue diagnosis of eight items for the five KMSs was unexpectedly low [63.8 \pm $10.1 \%(\mathrm{SD})]$ between the first and second tests, and it tended 


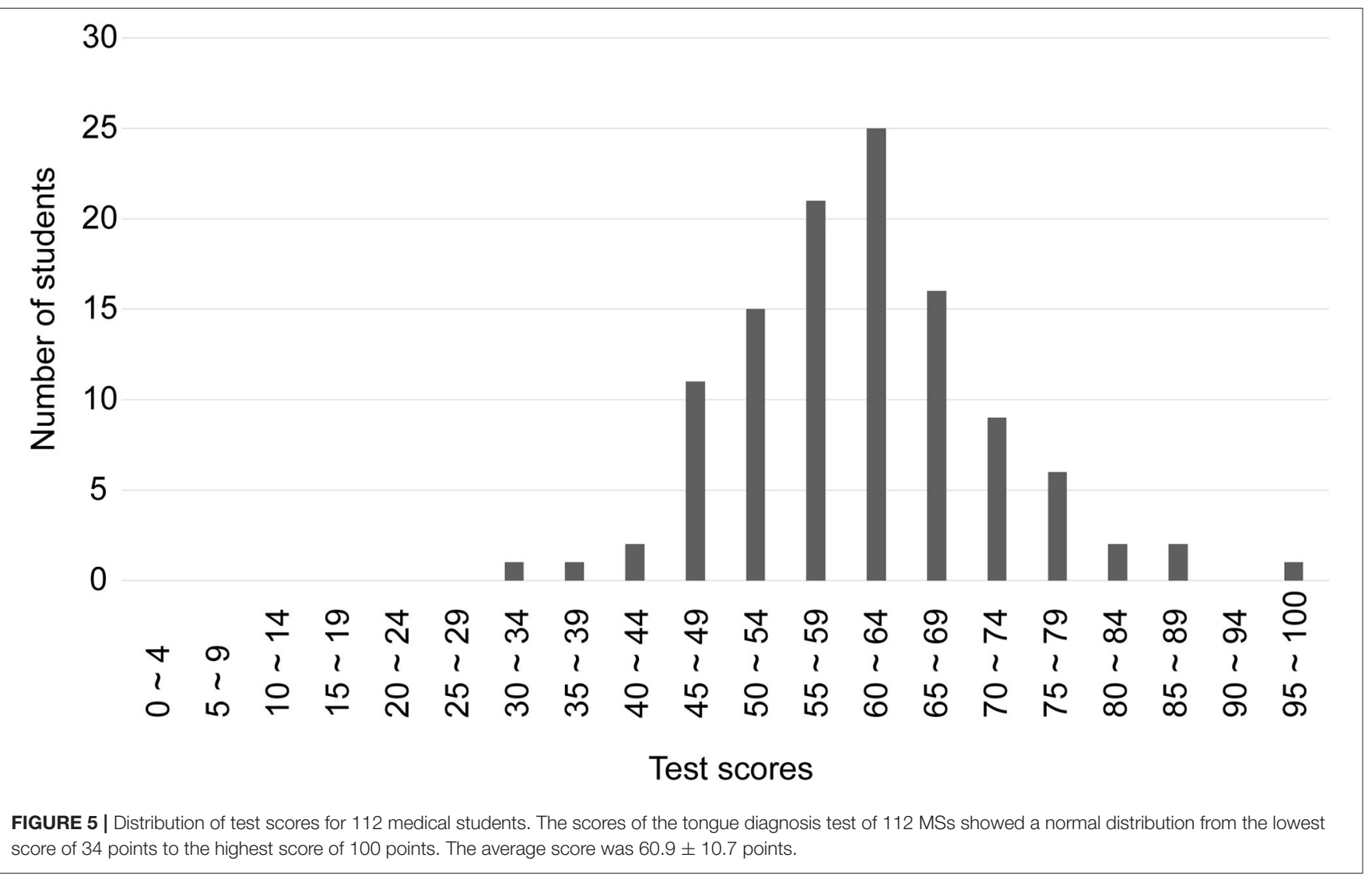

TABLE 4 | Comparison of mean diagnostic match rate for 10 tongue images between 112 MSs and five KMSs.

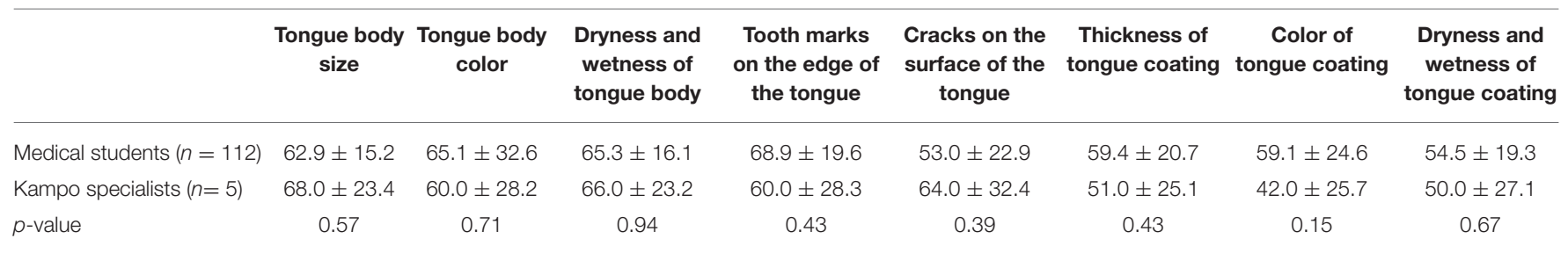

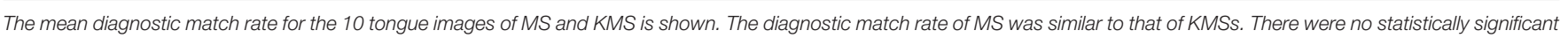
differences in any of the eight items. KMS, Kampo medicine specialists; MSs, medical students.

to be the lowest for an item related to color among the eight items. There are individual differences in the perception of colors, and the frequency of color blindness is present in $8 \%$ of men and $0.4 \%$ of women (36). Based on previous findings (36) and our present findings, we suggest that the ability to diagnose color is unstable. In addition, the ability to discriminate colors, as well as visual acuity, declines with age. In this study, the color discrimination ability and visual acuity of the evaluators were not examined, but the evaluators who exhibited extreme deterioration of visual acuity were not included. Oji et al. evaluated the color discrimination ability of 68 Kampo medical practitioners using the Farnsworth-Munsell 100 Hue test tongue color images and found that tongue color diagnosis significantly differed between subjects with $<10$ years of experience and $>10$ years of experience, and practitioners with $>10$ years of experience could maintain a consistent diagnosis of tongue color regardless of their age (37).

To the best of our knowledge, this is the first study to use e-learning for tongue examination education. To verify the educational usefulness of this system, 112 MSs who took a lesson on tongue diagnosis were administered a tongue diagnosis test. We found that the scores of the MSs were almost similar to those of the KMSs, indicating the high performance of our constructed e-learning test in evaluating the learning effect in tongue examination. In addition, we found that by taking a lesson on tongue diagnosis, it is possible for MSs to temporarily acquire diagnostic knowledge that is almost the same as that of a KMS. However, further studies may be necessary to verify whether this high ability for tongue diagnosis can be maintained for a long period of time. 


\section{CONCLUSION}

We constructed a high-quality tongue image database and developed a tongue diagnosis e-learning system. Since this system can repeatedly self-learn at any time and place, it is possible to acquire efficient tongue diagnosis techniques. This learning management system using information and communication technology is expected to contribute to the modernization and standardization of tongue examination education.

\section{DATA AVAILABILITY STATEMENT}

The raw data supporting the conclusions of this article will be made available by the authors, without undue reservation.

\section{ETHICS STATEMENT}

The studies involving human participants were reviewed and approved by the Ethical Review Committee at the Clinical Research Center of Yamaguchi University Hospital. The patients/participants provided their written informed consent to participate in this study. Written informed consent was obtained

\section{REFERENCES}

1. Reamy BV, Derby R, Bunt CW. Common tongue conditions in primary care. Am Fam Phys. (2010) 81:627-34.

2. Gaddey HL. Oral manifestations of systemic disease. Gen Dent. (2017) 65:239.

3. van der Waal I. Atlas of Oral Disease. A Guide for Daily Practice. Berlin: Springer (2016).

4. Bøhmer T, Mowé M. The association between atrophic glossitis and protein-calorie malnutrition in old age. Age Ageing. (2000) 29:4750. doi: 10.1093/ageing/29.1.47

5. Tahara T, Shibata T, Okubo M, Yoshioka D, Ishizuka T, Sumi K, et al. A case of Plummer-Vinson syndrome showing rapid improvement of dysphagia and esophageal web after two weeks of iron therapy. Case Rep Gastroenterol. (2014) 8:211-5. doi: 10.1159/000364820

6. Kobayashi A, Iwasaki H. Pernicious anemia presenting as glossitis. CMAJ. (2020) 192:E434. doi: 10.1503/cmaj.191331

7. Erriu M, Pili FMG, Cadoni S, Garau V. Diagnosis of lingual atrophic conditions: associations with local and systemic factors. A descriptive review. Open Dent J. (2016) 10:619-35. doi: 10.2174/1874210601610010619

8. Otsuka K. Kampo: A Clinical Guide to Theory and Practice. London: Singing Dragon (2017).

9. Dawes N. Fukushin and Kampo. Abdominal Diagnosis in Traditional Japanese and Chinese Medicine. London: Singing Dragon (2020).

10. Nathanaël L, Zhou GB, Prasher B, Mukerji M, Chen Z, Brahmachari SK, et al. Traditional knowledge-based medicine: a review of history, principles, and relevance in the present context of P4 Systems medicine. Prog Prev Med. (2017) 2:e0011. doi: 10.1097/pp9.0000000000000011

11. Rong L. A study of the changes in and issues of diagnosis of "normal tongue" in Chinese Medicine. Nihon Kenkyu. (2016) 53:231-52. doi: 10.15055/00006259

12. Solos I, LiangvY. A historical evaluation of Chinese tongue diagnosis in the treatment of septicemic plague in the pre-antibiotic era, and as a new direction for revolutionary clinical research applications. J Integr Med. (2018) 16:141-6. doi: 10.1016/j.joim.2018.04.001

13. Yu F, Takahashi T, Moriya J, Kawaura K, Yamakawa J, Kusaka K, et al. Traditional Chinese medicine and Kampo: a review from the distant past for the future. J Int Med Res. (2006) 34:231-9. doi: 10.1177/147323000603400301 from the individual(s) for the publication of any potentially identifiable images or data included in this article.

\section{AUTHOR CONTRIBUTIONS}

MS and NI collected tongue image data. MS, HO, and YH performed data analysis. MS, NI, HO, and YH contributed to conception and writing of the manuscript. $\mathrm{KT}, \mathrm{HN}$, and $\mathrm{KU}$ critically revised the manuscript. All authors contributed to the construction of database and e-learning system and approved the final manuscript.

\section{FUNDING}

This research was carried out using a research grant from the Japan Kampo Medicine Education Foundation in 2020.

\section{ACKNOWLEDGMENTS}

We would like to thank the Japan Kampo Medicine Education Foundation for their financial support. We would also like to thank Editage (www.editage.com) for English language editing.

14. Terasawa K. Evidence-based reconstruction of Kampo medicine: Part IIthe concept of Sho. Evid Based Comp Alternat Med. (2004) 1:11923. doi: 10.1093/ecam/neh022

15. Anastasi JK, Currie LM, Kim GH. Understanding diagnostic reasoning in TCM practice: tongue diagnosis. Altern Ther Health Med. (2009) 15:18-28.

16. Maciocia G. Tongue Diagnosis in Chinese Medicine. Revised ed. Seattle, WA: Eastland Press (1995).

17. Japan Council for Kampo Medical Education. Essential Lecture on Kampo Medicine. Tokyo: Yodosha (2020) (in Japanese).

18. Ko MM, Lee JA, Kang BK, Park TY, Lee J, Lee MS. Interobserver reliability of tongue diagnosis using traditional Korean medicine for stroke patients. Evid Based Comp Alternat Med. (2012) 2012:209345. doi: 10.1155/2012/209345

19. Kim M, Cobbin D, Zaslawski C. Traditional Chinese medicine tongue inspection: an examination of the inter- and intrapractitioner reliability for specific tongue characteristics. J Altern Comp Med. (2008) 14:52736. doi: 10.1089/acm.2007.0079

20. Oji T, Mamiki T, Mitani K, Ueda K, Nakaguchi T, Kainuma M, et al. Japanese literature survey of tongue findings for the purpose of creating a unified multicenter description of clinical tongue diagnoses. Kampo Med. (2014) 65:223-30. doi: 10.3937/kampomed.65.224

21. Hanawa $T$, Odaguchi $H$, Wakasugi A, Ito G, Oikawa T, Suzuki K, et al. Standardization of Kampo medical findings in a specialized Kampo medical service facility. Kampo Med. (2013) 64:344-51 (in Japanese). doi: 10.3937/kampomed.64.344

22. Arai M, Nakada Y. The actual conditions of traditional Japanese Kampo education in all the pharmacy schools in Japan: a questionnaire survey after the enforcement of the new national 2015 core curriculum. MC Comp Altern Med. (2018) 18:297. doi: 10.1186/s12906-018-2368-5

23. Lo LC, Chen YF, Chiang JY, Cheng TL, Damdinsuren N. Education of Chinese medicine tongue diagnosis by automatic tongue diagnosis system. Chin J Integr Med. (2015) 1-10. doi: 10.1007/s11655-015-2135-0

24. Tsai CC, Lo YC, Chiang JY, Sainbuyan N. Digital education and dynamic assessment of tongue diagnosis based on Mashup technique. Chin J Integr Med. (2017) 1-7. doi: 10.1007/s11655-016-2733-5

25. Nakaguchi T, Takeda K, Ishikawa Y, Oji T, Yamamoto S, Tsumura N, et al. Proposal for a new noncontact method for measuring tongue moisture to assist in tongue diagnosis and development of the tongue image analyzing 
system, which can separately record the gloss components of the tongue. Biomed Res Int. (2015) 2015:249609. doi: 10.1155/2015/249609

26. Zhang Q, Zhou J, Zhang B. Computational traditional Chinese medicine diagnosis: a literature survey. Comput Biol Med. (2021) 133:104358. doi: 10.1016/j.compbiomed.2021.104358

27. Zhang D, Zhang H, Zhang B. Tongue Image Analysis. Berlin: Springer (2017).

28. Wang X, Liu J, Wu C, Liu J, Li Q, Chen Y, et al. Artificial intelligence in tongue diagnosis: using deep convolutional neural network for recognizing unhealthy tongue with tooth-mark. Comput Struct Biotechnol J. (2020) 18:973-80. doi: 10.1016/j.csbj.2020.04.002

29. Feng C, Shao Y, Wang B, Qu Y, Wang Q, Li Y, et al. Development and application of artificial intelligence in auxiliary TCM diagnosis. Evid Based Comp Alternat Med. (2021) 2021:6656053. doi: 10.1155/2021/6656053

30. Jiang T, Hu XJ, Yao XH, Tu LP, Huang JB, Ma XX, et al. Tongue image quality assessment based on a deep convolutional neural network. BMC Med Inform Decis Mak. (2021) 21:147. doi: 10.1186/s12911-021-01508-8

31. Hu MC, Lan KC, Fang WC, Huang YC, Ho TJ, Lin CP, et al. Automated tongue diagnosis on the smartphone and its applications. Comput Methods Programs Biomed. (2019) 174:51-64. doi: 10.1016/j.cmpb.2017.12.029

32. Matos LC, Machado JP, Monteiro FJ, Greten HJ. Can traditional Chinese medicine diagnosis be parameterized and standardized? A narrative review. Healthcare. (2021) 9:177. doi: 10.3390/healthcare9020177

33. Ito A, Watanabe K, Fukuzawa Y, Mitani K, Fujimoto S, Matsuda T, et al. Development of Kampo(traditional Japanese medicine)e-learning program: evaluation of the flipped classroom for medical students. Med Educ Online. (2021) 26:1938504. doi: 10.1080/10872981.2021.1938504

34. Iizuka N, Usuku K, Nakae H, Segawa M, Wang Y, Ogashiwa K, et al. Web-based evaluation system to measure learning effectiveness in Kampo medicine. Evid Based Comp Alternat Med. (2016) 2016:2043535. doi: 10.1155/2016/2043535
35. Iizuka N, Nakae H, Segawa M, Tanaka K, Fujita Y, Ogihara H, et al. Web-based evaluation system for closing the education gap in Kampo medicine between facilities. Trad Kampo Med. (2019) 6:1-2. doi: 10.1002/tkm2.1207

36. Chan XB V, Goh SM S, Tan NC. Subjects with colour vision deficiency in the community: what do primary care physicians need to know? Asia Pac Fam Med. (2014) 13:10. doi: 10.1186/s12930-014-0010-3

37. Oji T, Namiki T, Nakaguchi T, Ueda K, Takeda K, Nakamura M, et al. Study of factors involved in tongue color diagnosis by kampo medical practitioners using the farnsworth-munsell 100 hue test and tongue color images. Evid Based Comp Alternat Med. (2014) 2014:783102. doi: 10.1155/2014/78 3102

Conflict of Interest: The authors declare that the research was conducted in the absence of any commercial or financial relationships that could be construed as a potential conflict of interest.

Publisher's Note: All claims expressed in this article are solely those of the authors and do not necessarily represent those of their affiliated organizations, or those of the publisher, the editors and the reviewers. Any product that may be evaluated in this article, or claim that may be made by its manufacturer, is not guaranteed or endorsed by the publisher.

Copyright (c) 2021 Segawa, Iizuka, Ogihara, Tanaka, Nakae, Usuku and Hamamoto. This is an open-access article distributed under the terms of the Creative Commons Attribution License (CC BY). The use, distribution or reproduction in other forums is permitted, provided the original author(s) and the copyright owner(s) are credited and that the original publication in this journal is cited, in accordance with accepted academic practice. No use, distribution or reproduction is permitted which does not comply with these terms. 